\title{
Capacité d'accueil des gravières de la plaine alluviale de la Garonne vis-à-vis du Grèbe huppé (Podiceps cristatus L.)
}

\author{
F. Santoul ${ }^{1}$ \\ J.-N. Tourenq ${ }^{1}$
}

Mots-clés : Podiceps cristatus, gravières, variables environnementales, capacité d'accueil.

Les activités humaines, à l'origine de la disparition de nombreuses zones humides naturelles, permettent la création de milieux aquatiques artificiels comme les lacs de barrage, les rizières ou les gravières. Dans la région Midi-Pyrénées, la création de plus en plus importante de gravières a permis l'installation et la nidification de plusieurs espèces d'oiseaux d'eau dont le Grèbe huppé (Podiceps cristatus L.). Cependant, après une phase de colonisation rapide, les effectifs stagnent à un niveau assez faible. Une étude des populations de Grèbe huppé a été réalisée pendant deux ans sur deux sites distincts de la plaine alluviale de la Garonne. L'analyse de la distribution des oiseaux en fonction des paramètres du milieu et au cours du temps a permis de mettre en évidence les facteurs qui régissent le stationnement des grèbes. En période de nidification, la présence d'îles et de végétation aquatique et riveraine sont des éléments très favorables. Les zones de pleine eau, en milieu suffisamment ouvert, sont aussi des lieux d'accueil favorables, notamment en période de mue. Un dérangement humain trop important est toujours préjudiciable. Dans des plans d'aménagement et de gestion des zones humides, la prise en compte de ces informations permettra une meilleure colonisation de ces milieux par les oiseaux.

Carrying capacity of gravel pits of the Garonne floodplain for the Great Crested Grebe (Podiceps cristatus L.)

Keywords : Podiceps cristatus, gravel pits, environmental factors, carrying capacity.

Human activities generate the destruction of natural wetlands, but also the creation of artificial ones e.g. rice fields, dam lakes or gravel pits. In the Midi-Pyrenees region (South West of France), the increasing number of gravel pits has allowed the colonisation and nesting of numerous species of water birds such as the Great Crested Grebe (Podiceps cristatus L.). However, after a fast colonisation by the gravel pits, their abundance seems to be low and stable. A study of Great Crested Grebe populations was carried out during two years on two distinct sites of the Garonne alluvial plain. Analysis of bird spatial distribution in relation with some environmental parameters has facilitated recognition of factors regulating grebe distribution. During the nesting period, the presence of quite and undisturbed areas (e.g. island on the lake) and a high riparian vegetation density are the most important factors. In the same way, large open water area constitute secure habitats, particularly during the moulting period. In contrast, human disturbance was always found prejudicial to the birds. These information will be useful for management purposes.

\section{Introduction}

En France, l'exploitation des granulats dans les plaines alluviales a fortement augmenté depuis une vingtaine d'années. L'accroissement des besoins et l'interdiction d'extraire dans le lit mineur des cours d'eau

1. Centre d'Ecologie des Systèmes Aquatiques Continentaux, UMR C5576 CNRS/UPS, Université Paul Sabatier 118, Route de Narbonne, F-31062 Toulouse Cedex.

E-mail : santoul@cict.fr sont à l'origine de cette hausse. Ces extractions entrâ̂nent la formation de nombreuses gravières. Ces milieux sont des systèmes limniques à part entière (Testard 1983), qui offrent de grande capacité d'accueil, et constituent des zones propices à la mise en place et l'évolution de la faune (Oiseaux, Batraciens, Poissons) et de la flore. Ainsi, nous assistons à la création de nouveaux milieux aquatiques à l'origine de phénomènes de colonisation d'un grand intérêt écologique (Frochot \& Godreau 1995).

La multiplication des gravières dans le bassin de la Garonne a permis l'installation et la nidification récente de nombreuses espèces d'oiseaux d'eau. Les oiseaux 
sont une composante à part entière des écosystèmes d'eau douce (lacs, rivières,..) mais leur rôle dans la chaîne trophique a souvent été négligé. En tant qu'homéothermes leur consommation en nourriture est relativement élevée. Ainsi, par exemple, un Grèbe huppé (Podiceps cristatus L.) avec un poids de $1 \mathrm{~kg}$ a une consommation annuelle 33 fois supérieure à celle d'un brochet (Esox lucius L.) de même poids (Cramp \& Simmons 1977).

Dans cette étude nous nous sommes intéressés à une espèce piscivore très fréquente dans les gravières de Midi-Pyrénées : le Grèbe huppé. Les gravières abritent $40 \%$ de la population régionale de Grèbe huppé (Joachim \& Bousquet, in litt.). En Angleterre, ce type de milieu existe depuis près d'un siècle et l'intérêt de ces plans d'eau de substitution pour les oiseaux d'eau est reconnu. Dès 1931 une réunion nationale sur le Grèbe huppé révèle l'importance de ces milieux pour cette espèce (Harrison \& Hollom 1932). En 1975, $60 \%$ de la population de Grèbes huppés d'Angleterre et du pays de Galles était sur les gravières et les réservoirs, comparé à $55 \%$ en 1965 (Hughes et al. 1979).

En France les effectifs en hivernage sont estimés à 33000 (Gillier et al. 2000). La région Midi-Pyrénées profite de l'expansion démographique de l'espèce qui niche régulièrement depuis une dizaine d'années seulement (Fontanet \& Bousquet 1997). Après une période de colonisation, les effectifs de Grèbes huppés semblent se stabiliser à un niveau assez faible (Comptage B:I.R.O.E, Association Régionale d'Ornithologie de Midi-Pyrénées (AROMP)). Très peu de gravières sont réhabilitées en zones naturelles, de nombreux sites sont reconvertis en bases de loisirs ou en lacs de pêche, source de multiples problèmes de dérangement et de qualité d'habitat pour les oiseaux. Les conditions d'accueil pour l'avifaune ne sont donc pas toujours optimisées.

Le but de cette étude est d'analyser les facteurs environnementaux - végétation, profondeur, dérangement... - qui interviennent dans le stationnement de Grèbes au niveau des gravières. L'étude a porté sur des gravières situées dans la plaine alluviale de la Garonne. Les résultats pourront être utilisés dans le cadre de la mise en place de plans d'aménagement de ces milieux.

\section{Matériel et méthodes d'étude}

\subsection{Sites d'étude (Fig. 1)}

Deux sites ont été choisis :

- Les gravières de Lavernose-Lacasse se situent à $31 \mathrm{~km}$ au Sud-Ouest de Toulouse dans le bassin versant de la Garonne, entre Saint-Gaudens et Toulouse.
Le site comprend de deux gravières, l'une gérée par une association de pêche, l'autre non aménagée.

- Les gravières de Saint Caprais se situent à $25 \mathrm{~km}$ au Nord de Toulouse sur la commune de Grenade. Elles sont localisées dans la plaine alluviale de la vallée de la Garonne près de son confluent avec l'Hers. Cette exploitation est en activité depuis 1979. Les quatre gravières suivies ont été remises en état mais ne sont pas aménagées.

Le secteur d'étude est soumis à un climat communément appelé "toulousain" voisin du climat continental, dominé par les vents d'Ouest. Il se caractérise par des hivers doux et humides et des étés chauds et secs avec parfois de violents orages.

La moyenne annuelle des précipitations est de $672,6 \mathrm{~mm}$. Les températures moyennes journalières varient de $5,4^{\circ} \mathrm{C}$ pour le mois le plus froid (janvier) à $21,9^{\circ} \mathrm{C}$ pour le mois le plus chaud (juillet) avec une température moyenne annuelle de $13,3^{\circ} \mathrm{C}$.

\subsection{Suivi des populations}

Des recensements hebdomadaires ont été effectués d'octobre 1996 à octobre 1998, soit 104 sorties par site en deux ans. La faible superficie et le caractère ouvert des sites permettent un dénombrement exhaustif des populations (Tamisier 1972). Les comptages sont effectués par un seul observateur grâce à des jumelles $8 \times 30$ et un télescope de $20 \times 60$. Le nombre et la position des Grèbes huppés sur chaque gravière est régulièrement noté.

Le Grèbe huppé étant essentiellement piscivore, l'ichtyofaune du milieu est donc un facteur essentiel à prendre en compte. Afin de pouvoir estimer ce peuplement au niveau de chaque gravière des pêches ont été réalisées à l'aide de filets en Nylon monofilament de maille 17 à $60 \mathrm{~mm}$.

\subsection{Paramètres environnementaux}

Différents paramètres du milieu ont été pris en compte :

Facteurs physiques et anthropiques : profondeur de l'eau - mesurée à l'aide d'un échosondeur - pente des berges, dérangement - présence ou absence des chemins aux abords des gravières - On distingue également, les zones de bordure des zones de pleine eau (Borowiec 1975).

Végétation rivulaire : les relevés ont été réalisés à deux niveaux :

- au niveau de la berge, 5 strates distinctes sont définies : terre-gravier ; pelouse ; herbacée ; strate arbustive; strate arborée ; 

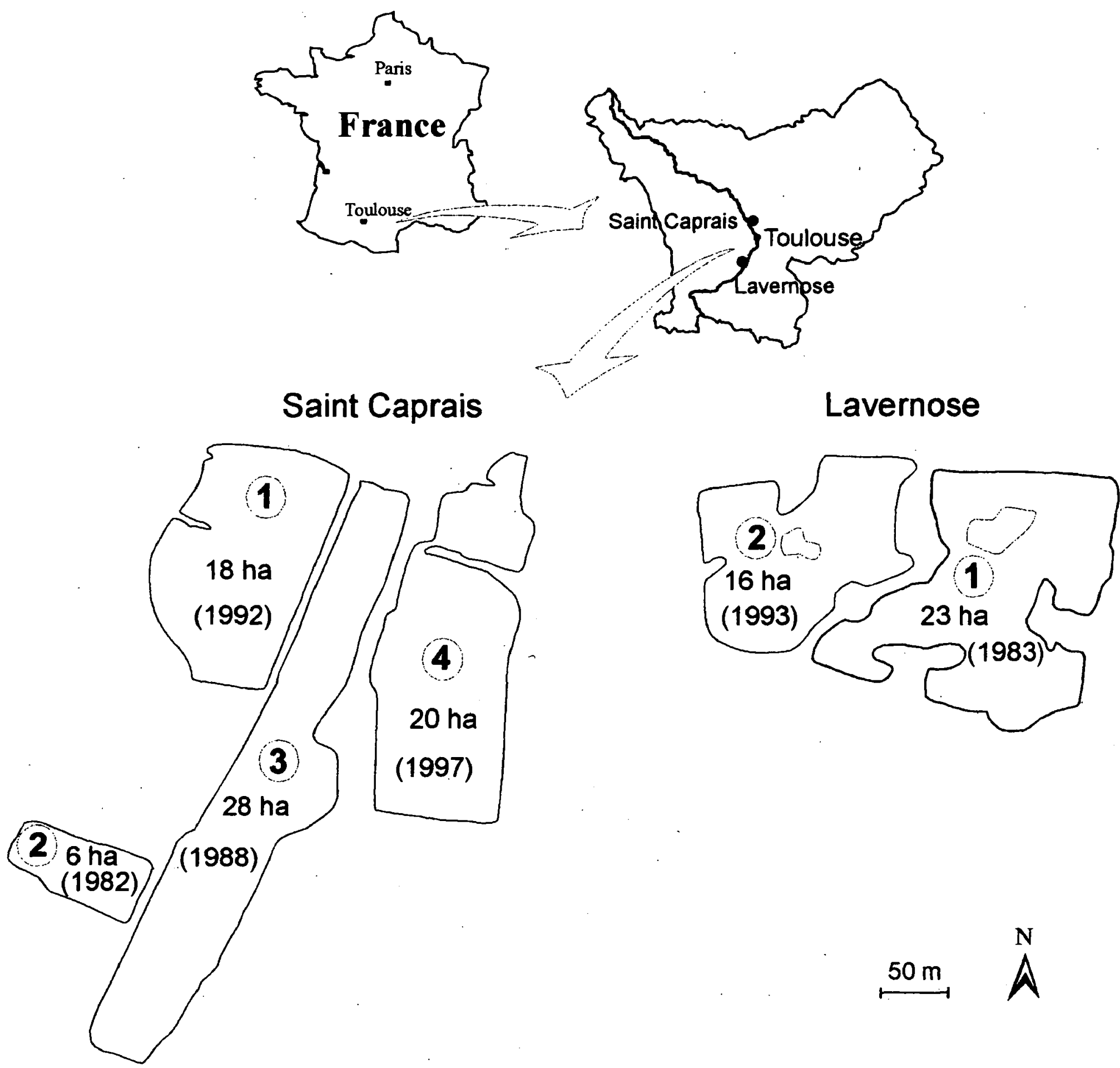

Fig. 1. Situation des sites d'étude.

Fig. 1. Localisation of the study sites.

- au niveau de la zone de marnage, le peu de végétation présente n'a permis d'établir que des classes de densité selon Bournaud et al. (1982) et Roche (1982).

Les relevés botaniques n'ont été effectués qu'une ou deux fois par gravière entre juin et août. Même si cela est insuffisant, ces relevés permettent de définir la nature et l'importance de la végétation pérenne qui se révèle prépondérante sur chaque site (Bournaud et al. 1980).

\subsection{Traitement des données}

Pour établir les relations espèce-variables, les variables quantitatives ont été découpées en modalités qualitatives ou semi-quantitatives : la "pente des berges" en trois modalités - < $10 \%, 10$ à $30 \%,>$ à $30 \%$ - ; la "végétation des rives" en cinq modalités terre-gravier ; pelouse ; strate herbacée ; strate arbustive ; strate arborée - ; la "végétation au niveau de la zone de marnage" en trois modalités - absence, faible, importante - ; pour les "chemins" deux modalités - 
présence, absence - ; pour la "profondeur de l'eau" - 0 à $1 \mathrm{~m}, 1$ à $3 \mathrm{~m}, 3$ à $4 \mathrm{~m}$-. Pour les "zones" deux modalités - bordure, pleine eau -. Pour les "îles" deux modalités - présence, absence -. "Année" : 1996/97, $1997 / 98$.

Il est extrêmement difficile de vouloir quantifier "le dérangement humain". Dans le cas très précis des gravières étudiées ici, la présence ou l'absence de chemins est le facteur le plus révélateur de ce dérangement. En effet, les pêcheurs se placent aux abords des chemins (zones de pêche autorisée), la chasse est interdite et les zones sans chemins sont privées. Cependant, le fait d'associer le dérangement humain à la présence ou absence de chemins n'est pas suffisant. Les dérangements ponctuels sont parfois très perturbants pour les oiseaux d'eau et ne sont pas pris en compte. La variable "dérangement humain" doit être considérée surtout comme une estimation générale de ces perturbations.

La variable "pente des berges" à Lavernose - une seule classe de pente $>30 \%$ - ni la variable "île" absente à Saint Caprais n'ont été analysées.

Afin d'étudier l'influence de tous les paramètres sur la répartition des grèbes au cours du temps, nous avons découpé chaque année en quatre périodes phénologiques. La période d'hivernage - octobre à février - ; les périodes de migration pré-nuptiale - mars-avril - et post-nuptiale - août-septembre - et la période de reproduction - mai à juillet-.

Au cours de chaque période des analyses statistiques ont été réalisées pour mettre en évidence l'influence des différents facteurs environnementaux.

La variabilité des densités d'effectifs en fonction des différents paramètres du milieu a été estimée par une analyse de variance (ANOVA) à un critère de classification (Wilkinson 1989). Lorsque les variations sont significatives $(\mathrm{P}<0,05 ; \mathrm{P}<0,01 ; \mathrm{P}<0,001)$, l'analyse a été complétée par un test de comparaison multiple des moyennes (Tukey-HSD). L'étude statistique a été réalisée à l'aide du logiciel Systat - 9.0.

\section{Résultats}

\subsection{Evolution des effectifs (Fig. 2)}

\section{- Gravières de Saint Caprais}

On observe des tendances similaires pour les deux années. Les effectifs les plus importants sont observés pendant la migration post-nuptiale. Cependant le nombre maximum d'oiseaux observés ne dépasse pas une quinzaine d'individus. Les effectifs sont faibles en hivernage, surtout en $97 / 98$.

\section{- Gravières de Lavernose}

On note également pour les deux années des évolutions semblables. Sur ces gravières les effectifs sont beaucoup plus stables au cours du temps. Le nombre d'individus - entre 4 et 6 - est peu important.

\subsection{Reproduction}

Les premières observations de jeunes sont : 8/07 en 1997, 26/05 en 1998 pour Saint Caprais, 30/05 en 1997, 22/05 en 1998 pour Lavernose.

8 nichées ont étés suivies au cours de ces deux années sur les deux sites - 4 à Saint Caprais ; 4 à Lavernose -, le nombre moyen de juvéniles à l'envol par couple est de 0,875 - 1,25 à Lavernose ; 0,5 à Saint Caprais-.

\subsection{Répartition (Tableau 1)}

- Gravières de Saint Caprais

En période d'hivernage, les Grèbes huppés sont préférentiellement en zone de pleine eau, et dans des zones de profondeurs comprises entre 3-4 $\mathrm{m}$ (test de Tuckey, $\mathrm{p}<0,001$ ).

Les observations sont identiques en période de migration pré-nuptiale. Deux facteurs supplémentaires interviennent en période de reproduction : la végétation riveraine et la pente des berges. Les Grèbes huppés se situent de manière significative près des strates arborées (test de Tuckey, $\mathrm{p}<0,05$ ), dans des zones de pente $<10 \%$ (test de Tuckey, $p<0,001$ ). Les facteurs corrélés positivement à la présence des grèbes, en période de migration post-nuptiale, sont les mêmes qu' en hivernage - zone de pleine eau à - 3-4 $\mathrm{m}$ -

\section{- Gravières de Lavernose}

De nombreux facteurs sont corrélés positivement avec la présence des Grèbes. On note que pour plusieurs facteurs il n'y a pas de variations selon les différentes périodes phénologiques.

La végétation de la rive et de la zone de marnage influence le stationnement des Grèbes. La strate herbacée de la rive et la végétation importante au niveau de la zone de marnage sont fréquentées préférentiellement (test de Tuckey, $\mathrm{p}<0,001$ ).

L'importance de la présence des îles a pu être testée sur ce site. Les Grèbes sont fortement liés $(p<0,001)$ à ce facteur. Les zones de pleine eau sont occupées en période de migration post-nuptiale $(\mathrm{p}<0,05)$.

Le rôle de sécurité, joué par les îles et les zones de pleine eau, est encore plus marqué à Lavernose, si on le compare à l'importance du dérangement humain $(\mathrm{p}<$ 0,001 ) près des berges. 


\section{Saint Caprais}

$\begin{array}{ccc}\text { migration } & \text { migration } \\ \text { pré nuptiale } & \text { post nuptiale }\end{array}$
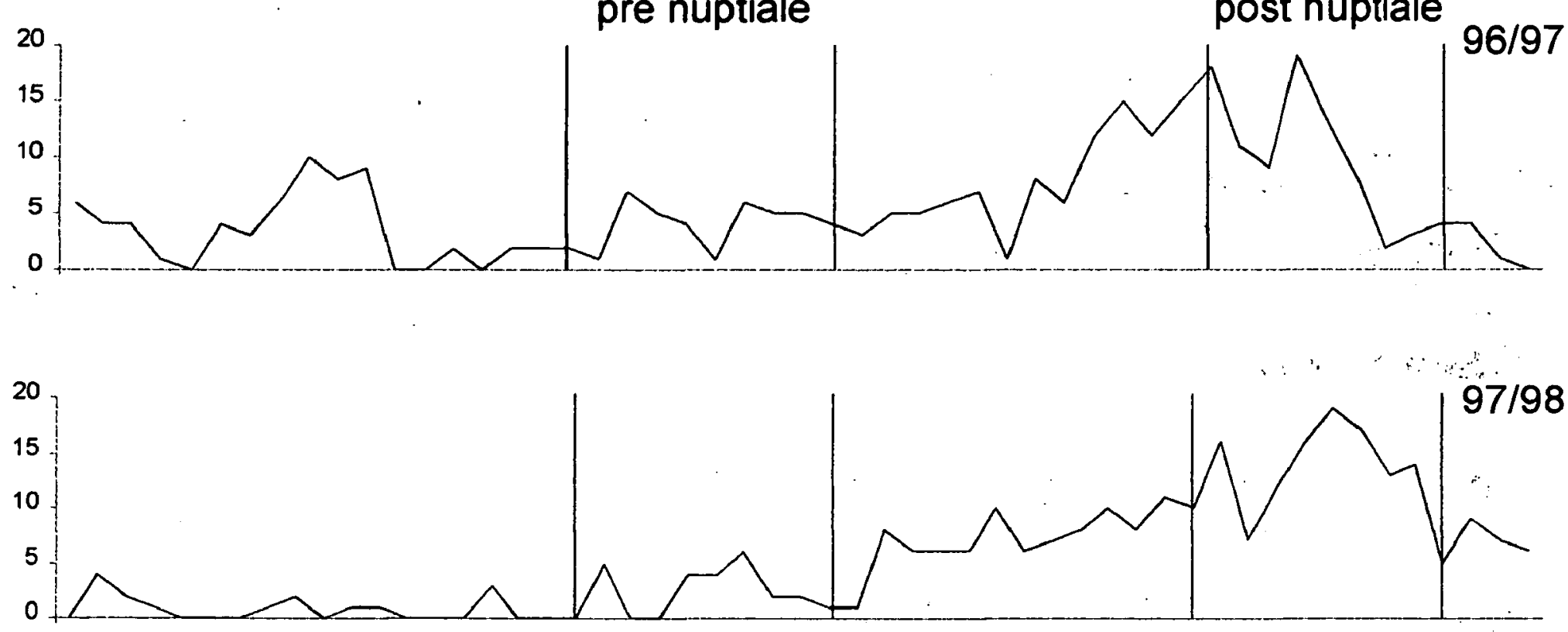

Lavernose

hivernage

migration reproduction

migration pré nuptiale
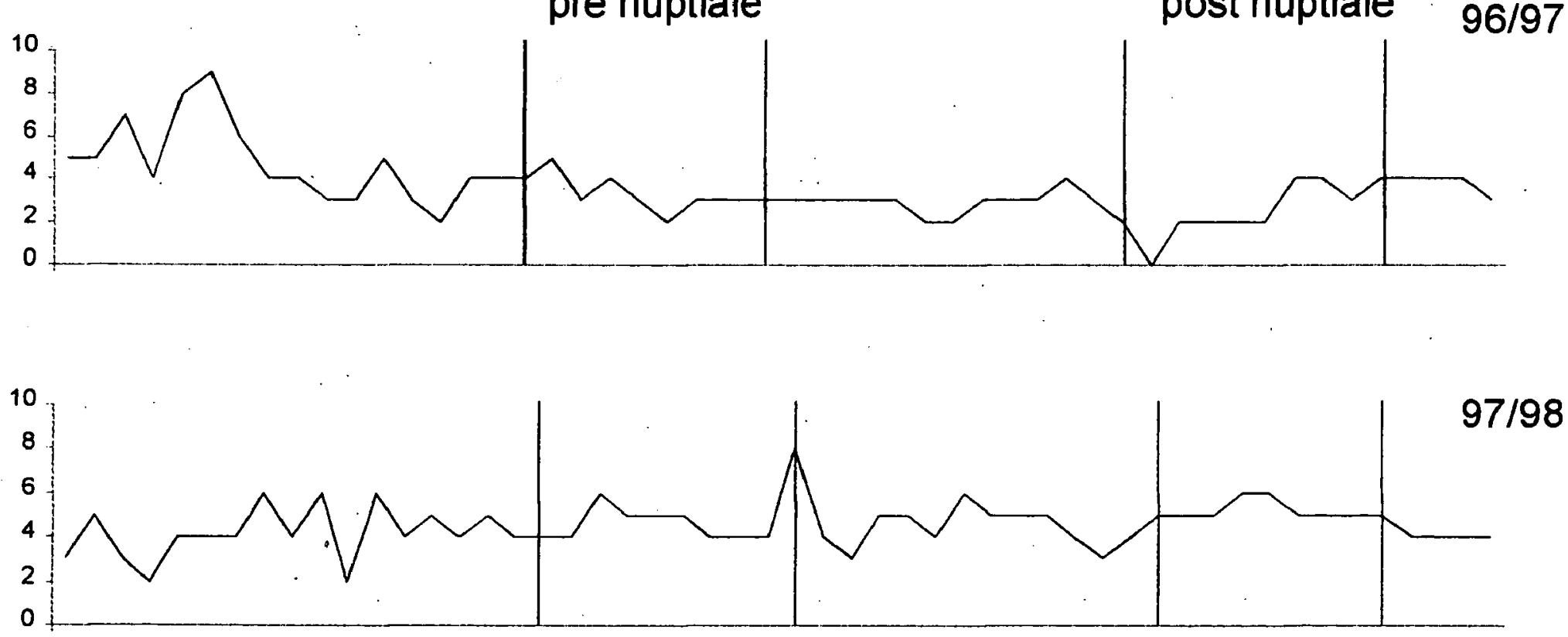

Fig. 2. Variation des effectifs de Grèbe huppé sur les sites de Saint Caprais et Lavernose.

Fig. 2. Evolution of the Great Crested Grebe population in the gravel pits of Saint Caprais and Lavernose. 
Tableau 1. Répartition du Grèbe huppé en fonction des paramètres du milieu.

Probabilité générale donnée par l'ANOVA : NS : $\mathrm{P}>0,05 ; *$ : $\mathrm{P}<0,05 ;{ }^{* *}: \mathrm{P}<0,01 ;{ }^{* * *}: \mathrm{P}<0,001$

( ) modalité concernée, Test de Tuckey (cf annexe)

Table 1. Distribution of the Great Crested Grebe according to the environmental parameters.

\begin{tabular}{|c|c|c|c|c|c|c|c|}
\hline Saint Caprais & Année & Zone & $\begin{array}{l}\text { Végétation de } \\
\text { la rive }\end{array}$ & $\begin{array}{l}\text { Végétation de la } \\
\text { zone de marnage }\end{array}$ & $\begin{array}{l}\text { Pente de } \\
\text { la berge }\end{array}$ & Profondeur & $\begin{array}{l}\text { Dérangement } \\
\text { humain }\end{array}$ \\
\hline Hivernage & NS & $\begin{array}{c}\star \star \star \\
\text { (Pleine eau) }\end{array}$ & NS & NS & NS & $\begin{array}{l}\star \star \star \\
(3-4 m)\end{array}$ & NS \\
\hline Migration pré-nuptiale & NS & $\begin{array}{c}\star \star \star \\
\text { (Pleine eau) }\end{array}$ & NS & NS & NS & $\begin{array}{l}\star \star \star \\
(3-4 m)\end{array}$ & NS \\
\hline Reproduction & NS & $\begin{array}{c}\star \star \star \\
\text { (Pleine eau) }\end{array}$ & $\begin{array}{c}\star \\
\text { (St. arborée) }\end{array}$ & NS & $\begin{array}{l}\star \star \star \\
(<5 \%)\end{array}$ & $\begin{array}{l}\star \star \star \\
(3-4 m)\end{array}$ & NS \\
\hline Migration post-nuptiale & NS & $\begin{array}{c}\star \star \star \\
\text { (Pleine eau) }\end{array}$ & NS & NS & NS & $\begin{array}{l}\star \star \star \\
(3-4 m)\end{array}$ & NS \\
\hline
\end{tabular}

\begin{tabular}{|c|c|c|c|c|c|c|c|}
\hline Lavernose & Année & Zone & $\begin{array}{l}\text { Végétation } \\
\text { de la rive }\end{array}$ & $\begin{array}{l}\text { Végétation de la } \\
\text { zone de marnage }\end{array}$ & Profondeur & Ile & $\begin{array}{l}\text { Dérangement } \\
\text { humain }\end{array}$ \\
\hline Hivernage & NS & NS & $\begin{array}{c}\star \star \star \\
\text { (St herbacée) }\end{array}$ & $\begin{array}{c}\star \star \star \\
\text { (Importante) }\end{array}$ & $\begin{array}{l}\star \star \star \\
(1-3 \mathrm{~m})\end{array}$ & $\begin{array}{c}\star \star \star \\
\text { (Présence) }\end{array}$ & $\begin{array}{c}\star \star \star \\
\text { (Absence de route) }\end{array}$ \\
\hline Migration pré-nuptiale & NS & NS & $\begin{array}{c}\star \star \star \\
\text { (St herbacée) }\end{array}$ & $\begin{array}{c}\star \star \star \\
\text { (Importante) }\end{array}$ & $\begin{array}{l}\star \star \star \\
(1-3 \mathrm{~m})\end{array}$ & $\begin{array}{c}\star \star \star \\
\text { (Présence) }\end{array}$ & $\begin{array}{c}\star \star \star \\
\text { (Absence de route) }\end{array}$ \\
\hline Reproduction & $\begin{array}{c}\star \\
(97 / 98)\end{array}$ & NS & $\begin{array}{c}\star \star \star \\
\text { (St herbacee) }\end{array}$ & $\begin{array}{c}\star \star \star \\
\text { (Importante) }\end{array}$ & $\begin{array}{l}\star \star \star \\
(1-3 \mathrm{~m})\end{array}$ & $\begin{array}{c}\star \star \star \\
\text { (Présence) }\end{array}$ & $\begin{array}{c}\star \star \star \\
\text { (Absence de route) }\end{array}$ \\
\hline Migration post-nuptiale & $\begin{array}{c}\star \\
(97 / 98)\end{array}$ & $\begin{array}{c}\star \\
\text { (Pleine eau) }\end{array}$ & $\begin{array}{c}\star \star \star \\
\text { (St herbacke) }\end{array}$ & $\begin{array}{c}\star \star \star \\
\text { (Importante) }\end{array}$ & $\stackrel{\star}{\star(1-3 \mathrm{~m})}$ & $\begin{array}{c}\star \star \star \\
\text { (Présence) }\end{array}$ & $\begin{array}{c}\star \star \star \\
\text { (Absence de route) }\end{array}$ \\
\hline
\end{tabular}

\subsection{Poissons (Tableau 2)}

De nombreux poissons ont été recensés sur les deux sites, parmi eux beaucoup de cyprinidés entre 5 et $15 \mathrm{~cm}$ qui sont des proies de prédilection pour les Grèbes (Sobczyk 1975). Des différences entre les gravières au niveau des communautés pisciaires sont notées avec quantitativement plus de poissons à Lavernose. Cependant toutes possèdent des effectifs pisciaires importants, on ne trouve pas de gravière pauvre en poisson qui serait alors moins prisée par les Grèbes.

\section{Discussion}

Les effectifs de Grèbes huppés sur les deux sites suivis restent assez faibles. Fontanet \& Bousquet (1997) ont signalé également sur des retenues artificielles de la région Midi-Pyrénées entre une et deux dizaines d'oiseaux. Ces effectifs sont assez stables ces six dernières années comme le montre au niveau des effectifs régionaux les recensements B.I.R.O.E : 1995 : 118, $1996: 325,1997: 341,1998: 292,1999: 231,2000$ : 397 (AROMP). Ils sont le reflet d'une évolution démographique stable et/ou de conditions.d'accueil pas tou- 
Tableau 2. Inventaire des poissons sur les sites de Saint Caprais et Lavernose.

Table 2. List of species found in the sites of Saint Caprais and Lavernose.

\begin{tabular}{llcc}
\hline Nom vernaculaire & Nom scientifique & Lavernose & Saint Caprais \\
\hline Ablette & Alburnus alburnus & + & + \\
Brème bordelière & Blicca bjoerkna & + & + \\
Carpe & Cyprinus carpio & + & + \\
Gardon & Rutilus rutilus & + & + \\
Rotengle & Scardinus erythrophthalmus & & + \\
Carassin & Carassius carassius & + & + \\
Poisson-chat & Ictalurus melas & + & + \\
Truite arc-en-ciel & Oncorhynchus myckiss & + & + \\
Perche soleil & Lepomis gibbosus & + & + \\
Black bass & Micropterus salmoides & + & + \\
Perche & Perca fluviatilis & + & + \\
Sandre & Stizostedion lucioperca & + & + \\
Brochet & Esox lucius & + & + \\
\end{tabular}

jours favorables. Les effectifs très stables notés au cours de la période d'étude sur les gravières de Lavernose, ajoutés à l'observation hebdomadaire du comportement des oiseaux - parade, construction du nid, élevage des jeunes, répartition au sein des gravières - permet de penser que les oiseaux observés sont sédentaires. Les mêmes tendances ont été notées à Puydarieux (Hautes-Pyrénées) et à Montbel (Ariège) (Fontanet \& Bousquet 1997).

Sur le site de Saint Caprais, les observations sont plus irrégulières. On constate cependant des fluctuations liées à des déplacements ponctuels d'individus certainement dans des gravières voisines. On note également une augmentation sensible pendant la période de migration post-nuptiale. Cette augmentation d'effectif de courte durée puisqu'elle disparaît en période d'hivernage, est probablement due à un événement du cycle biologique de l'oiseau : la mue. Chez le Grèbe huppé c'est un événement complexe dont la partie la plus importante (mue des rémiges) a lieu vers le mois d'octobre (Piersma 1988). Pendant cette période délicate de la vie de l'oiseau, les lieux ouverts sécurisants sont privilégiés. Les gravières de Saint Caprais offrent ces conditions pour le Grèbe huppé.

Sur les deux sites le Grèbe huppé se reproduit. Le nombre de couples observés reste faible avec un maximum de 3 couples en 1998 à Lavernose. Certains autres sites abritent également plusieurs couples nicheurs, c'est le cas du lac de Montbel, de Puydarieux et des retenues de Moissac-Saint-Nicolas (Tarn et Garon- ne) et de Cazères (Haute-Garonne) où entre 3 à 5 couples sont régulièrement recensés (Joachim, in litt.). Cependant, de nombreux sites de Midi-Pyrénées n'accueillent généralement qu'un seul couple (Joachim et al. 1997). Des aménagements simples, notamment la mise en place de palettes pour la nidification, ont augmenté le nombre de couples nicheurs du lac collinaire de Puydarrieux.

Selon les sites, les lieux de nidification sont différents. A Saint Caprais, les oiseaux nichent dans une petite ceinture de typhas (Typha latifolia) (nord de la gravière 4) et dans des branches plus ou moins immergées près des berges de la gravière 3. A Lavernose, toutes les nidifications ont été observées autour de l'île de la gravière 1 , également dans des branches à la surface de l'eau. Une seule tentative de construction du nid a été notée dans des branches près de la berge, mais elle n'a pas abouti. Le Grèbe huppé construit ainsi souvent son nid dans des branchages de saules mais il affectionne également des zones de roseaux ou de typhas (Mc Cartan \& Simmons 1956, Cramp \& Simmons 1977).

Concernant les premières observations de jeunes, les résultats diffèrent de ceux observés jusqu'à maintenant. En effet, les premières éclosions ont été signalées à Puydarrieux, la première décade de juin (Joachim et al. 1997), mais généralement les premiers jeunes sont seulement observés en juillet. Le marnage important des plans d'eau noyant la première ponte, les jeunes oiseaux observés en juillet sont issus d'une ponte de rem- 
placement. Sur les gravières de Saint Caprais en 1998 et de Lavernose en 1997 et 1998, les premières éclosions recensées à la fin du mois de mai correspondent à des succès des premières pontes. Le marnage moins important de ces gravières par rapport à d'autres lacs collinaires peut expliquer cette réussite.

Sur les gravières de Lavernose en 1998, des éclosions observées le 7/08/1998 correspondaient à une deuxième ponte d'un couple après le succès de la première. La saison de reproduction des Grèbes huppés est très étalée (Piette \& Ruwet 1990).

Le nombre moyen de juvéniles à l'envol par couple à Lavernose $(1,25)$ est comparable à celui observé sur une population de Grèbes au Royaume Uni $(1,3)$ moyenne obtenue à partir de 431 couples sur différents milieux (Cramp \& Simmons 1977). D'autres études ont montré que le nombre moyen de juvéniles à l'envol par couple peut être beaucoup plus élevé. Ainsi, dans des gravières de Belgique réaménagées en lac de pêche, le nombre moyen de juvéniles à l'envol par couple est de 2,3 et 2,88 pour les deux années de suivis (Duyck 1993). Dans le Nord de la France, en Picardie, une étude menée sur quatre ans a signalé un nombre moyen comparable de juvéniles à l'envol par couple de 2,39 (Commecy 1986).

A Saint Caprais le nombre moyen de juvéniles est beaucoup plus faible $(0,5)$. Toutes les observations effectuées ne nous ont pas permis d'observer d'acte de prédation sur les poussins. Le brochet (Esox lucius L.) est souvent cité comme le principal prédateur (Mc Cartan \& Simmons 1956, Cramp \& Simmons 1977). Ce poisson n'a pas été capturé sur le site de Saint Caprais (Tableau 2). Les causes de disparition des poussins à Saint Caprais peuvent être attribuées à la prédation par le Busard des roseaux (Circus aeruginosus L.) (Fontanet, in litt.), Goéland leucophée (Larus cachinnans L.). Nous avons pu observer un cas très rare de prédation de Grèbe huppé immature (mais de taille proche de l'adulte) par un Héron cendré (Ardea cinerea L.). D'après la bibliographie, une seule observation similaire a été effectuée (Besson 1995). Cette observation est considérée c̀omme anecdotique. De plus, il est important de préciser que les résultats concernant le nombre moyen de juvéniles à l'envol par couple ne prend en compte que peu d'individus. Aussi, les résultats obtenus sont faibles et doivent être nuancés. Une période d'étude plus longue permettrait de préciser ces tendances.

L'analyse des relations espèces/variables du milieu, nous permet de mettre en évidence selon les différentes périodes de l'année, les facteurs qui influencent la distribution des Grèbes huppés dans le milieu. Le
Grèbe n'utilise pas les différentes zones du lac de manière uniforme (Sobczyk 1975).

Les lacs possédant une ceinture de végétation assez large mais peu dense ainsi qu'une surface en eau étendue avec une profondeur pas trop faible sont des biotopes préférés du Grèbe huppé (Cramp \& Simmons 1977).

Pour les gravières de Saint Caprais, quelle que soit la période de l'année, les oiseaux colonisent préférentiellement les zones de pleine eau. Ces zones ouvertes offrent les meilleures conditions de sécurité aux Grèbes : peu enclins au vol, ils préfèrent s'éloigner des sources de danger potentiel en plongeant.

Les profondeurs - 3-4 m sont également les plus fréquentées. L'impact de la profondeur des gravières sur le Grèbe huppé dans notre région doit être nuancé. En effet la profondeur des gravières dans le bassin de la Garonne n'excède généralement pas - $4 \mathrm{~m}$, or cette profondeur n'est pas un facteur limitant pour le Grèbe qui peut plonger plus profond pour rechercher sa nourriture (Juillard 1992).

En période de reproduction, deux facteurs supplémentaires sont corrélés positivement à la présence des Grèbes : la végétation de la rive et la pente des berges. Les zones boisées avec une pente faible sont les plus fréquentées. Les zones boisées utilisées par les Grèbes à Saint Caprais offrent les conditions de sécurité pour les nids, et les matériaux nécessaires à leur construction.

En période de migration post-nuptiale, on retrouve les mêmes facteurs - profondeur, zone de pleine eau qu'en période d'hivernage. Comme nous l'avons signalé précédemment les effectifs plus importants pendant l'hiver sont certainement dûs à des oiseaux venant avant leur période de mue. Dans ces conditions, les zones de pleine eau trouvent tout leur sens comme zone de sécurité.

Au niveau des gravières de Lavernose, les Grèbes se situent près de l'île ou en zone de pleine eau en période de migration post-nuptiale. La végétation importante au niveau de l'île, aussi bien pour la rive, que pour la zone de marnage leur permet de s'abriter et de construire leur nid.

Lors de la deuxième année, le nombre d'oiseau recensé est plus important en période de reproduction et en période de migration post-nuptiale, la reproduction ayant été plus importante. Cependant, les jeunes ne semblent pas rester sur le site.

L'analyse des observations de ces deux sites permet de mettre en évidence les principaux facteurs qui interviennent dans le stationnement du Grèbe huppé sur les 
gravières du bassin de la Garonne. Sur les gravières non aménagées de Saint Caprais, la population de Grèbe augmente en période de migration post-nuptiale; un rassemblement d'oiseaux pendant la période de mue parait l'explication la plus vraisemblable. Nous avons vu dans ce cas limportance des zones de pleine eau.

L'emplacement du site est également un facteur à ne pas oublier. Les gravières de Saint Caprais, situées au confluent de l'Hers et de la Garonne, ont une position privilégiée par rapport aux mouvements de migration des oiseaux.

En période de reproduction, la végétation arbustive ou arborée joue un rôle important. De ce fait les plantations réalisées pour le lac de pêche à Lavernose sont un atout. Le nombre de couples nicheurs est plus élevé sur ce site. A Saint Caprais la végétation arbustive peu représentée est l'un des principaux facteurs limitant pour la reproduction du Grèbe. L'absence d'îles est également préjudiciable. Le Grèbe huppé a un comportement territorial, en Grande Bretagne une moyenne de un couple pour $3500 \mathrm{~m}^{2}$ a été calculée (Harrison \& Hollom 1932). Cependant dans des lieux où les effectifs sont importants, les Grèbes peuvent se regrouper en colonies mixtes avec des mouettes ou en colonies monospécifiques (Goc 1986). Dans la région Midi-Pyrénées on note un comportement territorial des Grèbes, qui limite les possibilités d'accueil des sites et tout particulièrement ceux d'une faible superficie.

A Lavernose, on peut noter que toutes les reproductions avec succès ont lieu aux abords de l'île, ces zones offrant des conditions de sécurité plus importantes. Si l'on considère l'important facteur de dérangement lié à ce site. L'aménagement de ce site pour la pêche entraîne une augmentation accrue des visites. On note ainsi l'absence ou l'échec de nidification près des berges (excepté celles de l'île), le dérangement est la cause principale de ces échecs. Les Grèbes peuvent tolérer une présence humaine mais des dérangements importants peuvent entraîner un abandon du nid avec, le cas échéant, une ponte de remplacement (Keller 1989, Duyck 1993). La présence de chiens est, d'après nos observations sur le site, le facteur le plus perturbant pour les oiseaux.

Les pêches réalisées sur les différentes gravières montrent que le facteur nutrition n'est pas un limitant. Sur les sites étudiés, les poissons et notamment les cyprinidés capturés préférentiellement par les Grèbes sont suffisamment nombreux (Ulenaers \& Van Vessem 1994).

\section{Conclusion}

Le Grèbe huppé a profité de la création de milieux artificiels et des gravières en particulier pour s'installer. En Midi Pyrénées, les populations restent cependant assez faibles. Les oiseaux occupent des sites où les conditions d'accueil ne sont guère optimisées. L'étude réalisée nous a permis de mettre en évidence les facteurs limitants, notamment en période de reproduction. Des aménagements - création d'îlots,... - et certaines mesures - limitation du dérangement - permettraient d'augmenter la capacité d'accueil de ces sites. Cependant de telles mesures ne sont envisageables que si une mise en valeur biologique des gravières est entreprise. Or, à l'heure actuelle, ces milieux sont encore trop rares au niveau régional pour permettre un bon accueil des populations aviaires.

\section{Travaux cités}

Besson J-M. 1995. - Héron cendré prédateur de jeunes Grèbes huppés (Podiceps cristatus). Nos oiseaux, $42: 430$.

Borowiec E. 1975. - Food of coot (Fulica atra L.) in different phenological periods. Polskie Archivum Hydrobiologii, 22 (2) : 157-166.

Bournaud M., Ledant J.P., Broyer J. \& Richoux M. 1980. - Influence des paramètres physionomiques du milieu étang sur la distribution des oiseaux en période de nidification. Le Bièvre, 2 (1) : 25-47

Bournaud M., Ledant J.P., Broyer J. \& Richoux M. 1982. — L'espace étang dans ses rapports avec l'avifaune en période de nidification. Bull. Ecol., 13 (2) : 125-144.

Commecy X. 1986. - Eco-ethologie du Grèbe huppé en Picardie. L'Avocette, $10: 5-29$.

Cramp S. \& Simmons K.E.L. 1977. - Birds of the western Paleartic, Vol. 1. Oxford University Press, Oxford.

Duyck B. 1993. - La nidification du Grèbe huppé (Podiceps cristatus) sur les carrières innondées du Tournaisis en 1991-1992. Aves, 30 (2) : 141-144.

Fontanet M. \& Bousquet J.F. 1997 - in Joachim J., Bousquet J-F \& Faure Ch. (1997). Atlas des oiseaux nicheurs de Midi-Pyrénées, Association Régionale Ornithologique de Midi-Pyrénées : 262p

Frochot B. \& Godreau V. 1995. - Intérêt écologique des carrières, terrils et mines. Natures-Sciences-Sociétés, Hors Série : 66-7.6.

Gillier J-M., Maheo R. \& Gabillard F. 2000. -Les comptages d'oiseaux d'eau hivernant en France : actualisation des connaissances, effectifs moyens, critères numériques d'importance internationale et nationale. Alauda, 68 (1): 45-54.

Goc M. 1986. - Colonial versus territorial breeding of the great crested Grebe Podiceps cristatus on Lake Druzno. Acta ornithologica, 22 (2) : 95-141.

Harrison T.H. \& Hollom P.A.D. 1932. - The great crested Grebe enquiry 1931. British Birds, 26 ; 62-92, 102-131, 142-155, 174-195.

Hughes S.W.M., Bacon P. \& Flegg J.J.M. 1979. - The 1975 census of the great crested Grebe in Britain. Bird Study, 26 (4) : 213-226.

Joachim J., Bousquet J-F. \& Faure C. 1997. - Atlas des oiseaux nicheurs de Midi-Pyrénées, Association Régionale Omithologique de Midi-Pyrénées, 262p.

Juillard M. 1992. - Chasse en profondeur pour un Grèbe huppé. Nos oiseaux, 41 (2) : $111 \mathrm{p}$. 
Keller V. 1989. - Variations in the response of great crested Grebe (Podiceps cristatus) to human disturbance a sign of adaptation. Biological Conservation, 49 (1) : 31-46.

Mc Cartan. \& Simmons K.E.L. 1956. - Territory in the great crested Grebe Podiceps cristatus re-examinated. lbis, $98: 370-378$.

Piersma T 1988. - The annual moult cycle of great crested Grebes. Ardea, $76: 82-95$.

Piette V. \& Ruwet J-C. 1990. - Profils comportementaux, individuels et de couple, au sein d'une population nidificatrice de Grèbes huppés Podiceps cristatus L. Cah. Ethol. Appl., 10 (1) : 1-86.

Roche J. 1982. - Structure de l'avifaune des étangs de la plaine de Saône : influence de la superficie et de la divẹrsité végétale. Alauda, 3: 193-215.
Sobczyk R. 1975. - Great crested grebes (Podiceps cristatus L.) on some of the lakes in the Mazurian lakeland. Polskie Archivum Hydrobiologii, 22 (2), 181-194.

Tamisier A. 1972. - Etho-écologie des sarcelles d'hiver (Anas c. crecca $\mathrm{L}$.) pendant leur hivernage en camargue. Thèse d'état, Université de Montpellier : $157 \mathrm{p}$.

Testard P. 1983. - Le lac de Créteil (Région parisienne) : un lac de sablière peu profond. in Problèmes d'écologie. Ecosystèmes limniques. Masson, Paris.

Ulenaers P. \& Van Vassen J. 1994. — Impact of great crested Grebes (Podiceps cristatus L.) on fish ponds. Hydrobiologia, 279/280 : 353-366.

Wilkinson L. 1989. - Systat : the system for statistics. Evanston, Illinois : systat INC. : $638 \mathrm{p}$.

Annexe. Tests de Tuckey : NS : $\mathrm{P}>0,05 ; *: \mathrm{P}>0,05 ;{ }^{*} \mathrm{P}<0,01 ; * * *: \mathrm{P}<0,001$.

Appendix. Tuckey tests.

\section{Saint caprais}

\section{Profondeur}

(hiv, pré, repro, post)

\begin{tabular}{|c|c|c|}
\hline 1 & 2 & 3 \\
\hline 1 & NS & $\approx * \pi$ \\
\hline 2 & & $\pi \star \star \star *$ \\
\hline
\end{tabular}

\section{Végétation de la rive} (repro)

\begin{tabular}{|c|c|c|c|c|}
\hline 1 & 2 & 3 & 4 & 5 \\
\hline 1 & NS & NS & NS & $\star \star$ \\
\hline 2 & & NS & NS & $\star$ \\
\hline 3 & & & NS & $\star$ \\
\hline 4 & & & & NS \\
\hline
\end{tabular}

\section{Pente} (repro)

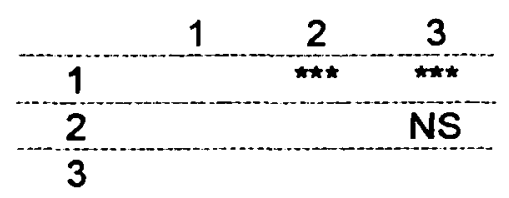

\section{Lavernose}

Végétation dè la zone de marnage (hiv)

\begin{tabular}{|c|c|c|}
\hline 1 & 2 & 3 \\
\hline 1 & $\star$ & 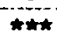 \\
\hline 2 & & $* * \star$ \\
\hline
\end{tabular}

Végétation de la zone de marnage (pré, repro, post)

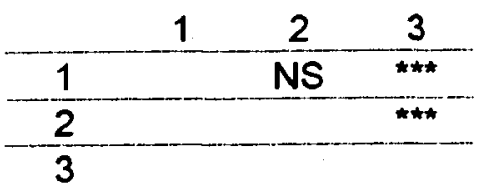

\section{Végétation de la rive} (hiv)

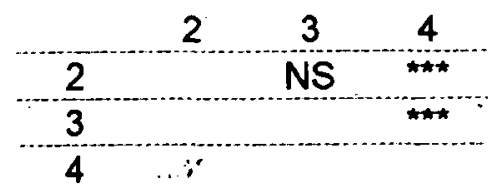

(pré)

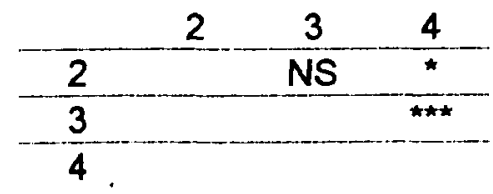

(repro, post)

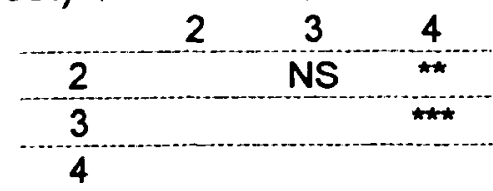

